\title{
DINÁMICA POBLACIONAL DE Cortaritermes fulviceps (SILVESTRI, 1901) (ISOPTERA: TERMITIDAE)
}

\author{
Giovana Annoni ${ }^{1}$, Juan Manuel Coronel $^{1}$, Enrique Rafael Laffont ${ }^{1}$ \\ ${ }^{1}$ Laboratorio de Biología de los Invertebrados. Universidad Nacional del Nordeste. Facultad de Ciencias Exactas y Naturales y \\ Agrimensura. Av. Libertad 5470. CP 3400. Corrientes, Argentina. E-mail: erl@exa.unne.edu.ar
}

\section{RESUMEN}

Se analizaron las poblaciones de 16 nidos de Cortaritermes fulviceps procedentes de diferentes localidades de la Provincia de Corrientes (Argentina) en las cuatro estaciones del año. Se extrajeron 4 nidos por cada estación. Se registró la presencia de reproductores en 7 de los nidos analizados. Las castas obreras, soldados e inmaduros blancos estuvieron presentes en todas las estaciones, no así los soldados blancos y pre-alados. La razón del total de obreras: soldados se mantuvo constante durante todo el año con una proporción de 6:1, excepto en primavera donde se registró un descenso a 3:1. Se registraron correlaciones positivas entre el total de obreras y soldados y entre inmaduros blancos y pre-alados, mientras que las correlaciones resultaron negativas entre los inmaduros blancos con las obreras y soldados, y entre pre-alados con obreras y soldados. Los pre-alados fueron encontrados en las 4 estaciones del año. Hubo diferencias significativas entre las estaciones en las proporciones de todas las castas, a excepción de las obreras menores. No se registraron diferencias significativas entre estaciones en el volumen de las poblaciones analizadas, al igual que entre el número de individuos estimados por termiteros.

Palabras claves: nidos, población, termites

\section{DINÂMICA POPULACIONAL DE Cortaritermes fulviceps (SILVESTRI, 1901) (ISOPTERA: TERMITIDAE)}

\section{RESUMO}

Foram analisadas as populações de 12 ninhos de Cortaritermes fulviceps, oriundos de diferentes localidades da provincia de Corrientes, Argentina, nas quatro estações do ano. Foram coletados 4 ninhos em cada estação. Registrou-se a presença de reprodutores em 7 dos ninhos analisados. As castas de operários, soldados e imaturos brancos foram observadas em todas as estações, o que não ocorreu com as de soldados brancos e pré-alados. A proporção de 6:1 do total de operários por soldados se manteve constante durante todo o ano, exceto na primavera quando caiu para 3:1. Registraram-se correlações positivas entre o total de operários e de soldados e entre imaturos brancos e pré-alados, enquanto que foram negativas as correlações entre imaturos brancos com operários e soldados, e entre os pré-alados com operários e soldados. Os pré-alados foram encontrados nas quatro estações do ano. Foram observadas diferenças significativas entre as estações nas proporções de todas as castas, exceto na dos operários menores. Não se registraram diferenças significativas entre as estações no volume das populações analisadas, bem como no número estimado de indivíduos por termiteiros.

Palavras-chave: ninhos, população, térmitas 


\section{INTRODUCCIÓN}

El orden Isoptera constituye uno de los grupos de artrópodos más abundantes en los ecosistemas terrestres de regiones tropicales y subtropicales de todo el mundo, $\mathrm{y}$ las especies que lo integran han sido reconocidos como los principales agentes que se ocupan de los procesos de descomposición de materia orgánica y reciclado de nutrientes en los ecosistemas de ésas regiones, actuando como especies claves en algunos de ellos (REDFORD, 1984; JOUQUET et al., 2004). Sin embargo, y a pesar de los importantes procesos en los que intervienen las termites en ecosistemas terrestres, en algunas especies se sabe muy poco sobre aspectos de su dinámica poblacional (MARTIUS, 1994; KORB \& LINSENMAIR, 2001; CORONEL \& LAFFONT, 2010; ANNONI et al., 2011).

Las proporciones de castas y fluctuaciones en la composición de las colonias han sido analizadas por diversos autores. Bodot (1969) establece que las variaciones en la composición de las colonias se deben a dos factores principales. El primero relacionado con el envejecimiento de las colonias donde diferencia tres períodos, una fase juvenil donde solo se encuentran neutros (obreras y soldados), una fase de madurez con producción regular de imagos, y una fase senil donde la producción de imagos declina. El segundo factor de variación obedece a un ciclo estacional regido por los cambios de temperatura en zonas templadas y por el régimen de lluvias en las zonas tropicales.

Las especies de este orden viven en sociedades integradas por un sistema de castas morfológica y funcionalmente bien definidas. Este sistema de castas constituye la infraestructura funcional de la colonia y los datos sobre las proporciones y rango de variación son fundamentales para entender la dinámica ecológica, fisiológica y reproductora de la especie (THORNE,
1985), para estimar la biomasa de termites por hectárea (HAVERTY \& NUTTING, 1975) y establecer la diferencia entre las especies morfológicamente similares (BAGINE et al., 1989).

Las nidificaciones de los isópteros, si bien pueden variar según la especie y el ambiente, se clasifican en dos grupos (BARONI-URBANI et al., 1978), el primero incluye a las especies que nidifican en los árboles y el segundo a aquellos que lo hacen en el suelo. Cortaritermes fulviceps se incluye en el segundo grupo; sus nidos, descriptos en detalle por Silvestri (1903) y Talice et al. (1969), Torales (1998) y Coronel y Laffont (2010) forman montículos de pequeño a mediano tamaño construidos con tierra una parte epígea e hipógea bien desarrolladas.

Si bien Cortaritermes fulviceps es una especie de muy amplia distribución en Argentina y países limítrofes (CONSTANTINO, 1998; TORALES et al., 2005; TORALES et al., 2008) a las contribuciones ya mencionadas de Silvestri (1903), Talice et al. (1969) y Torales (1998), sobre los diferentes aspectos de su biología, se le agrega una reciente publicación de Coronel y Laffont (2010) sobre la disposición espacial de los termiteros de esta especie y de Annoni et al. (2011) sobre las variaciones morfométricas en la casta obrera, desconociéndose hasta el momento, las proporciones de las castas que componen sus colonias y sus variaciones a lo largo del ciclo anual. Sin bien el artículo de Laffitte de Mosera et al., 1979, describe la composición de las poblaciones, considerando la presencia o ausencia de castas a lo largo del ciclo anual, pero sin analizar las proporciones de las diferentes castas. En este artículo se pretende llenar ese vacío aportando datos cuantitativos y su variación a lo largo del ciclo anual. 


\section{MATERIALES Y MÉTODOS}

Para el estudio de la dinámica poblacional de Cortaritermes fulviceps (Isoptera, Termitidae), se analizaron 4 nidos por cada estación, procedentes de Laguna Pampín (2729' 05,61”S 5844'44,88”O), Av. Maipú y Ruta $12 \quad\left(27^{\circ} 31^{\prime} 58,40^{\prime \prime}\right.$ S $58^{\circ} 46^{\prime} 25,33^{\prime \prime}$ ) $)$, y paraje El Perichón $\left(27^{\circ}\right.$ $25^{\prime} 38,70^{\prime \prime}$ 'S 58 44'38,00"O) de la ciudad de Corrientes (Dpto. Corrientes) y la localidad Mburucuyá (Dpto. Mburucuyá) $\left(28^{\circ} 01^{\prime} 08,48^{\prime \prime} \mathrm{S} \quad 58^{\circ} 01^{\prime} 42,69^{\prime \prime} \mathrm{O}\right)$ de la Provincia de Corrientes (Tabla 1). Se registraron las dimensiones $(\mathrm{cm}) \mathrm{y}$ características de los mismos, para luego ser extraídos utilizando una pala y depositados en el interior de bolsas plásticas para su traslado al laboratorio. El volumen de los termiteros se estimó por comparación con la mitad de un elipsoide, según la siguiente fórmula propuesta por Lefeuve (1987) para nidos epigeos:

$$
\frac{4 / 3 \pi \cdot(1 / 2 \mathrm{~L}) \cdot(1 / 2 \mathrm{~W})^{2}}{2}
$$

Donde $\mathrm{L}=$ altura + profundidad del nido $\mathrm{y}$ $\mathrm{W}=$ diámetro.

La disección de los montículos y la separación de las termites se llevó a cabo utilizando una combinación de la metodología de Darlington (1984) y Thorne (1985). Los nidos fueron fragmentados en pequeños trozos, el sector en que se localizó la pareja real fue cuidadosamente revisado, y una vez separados los reproductores se fijaron inmediatamente en FAA (formol + alcohol + ácido acético). El resto de la población fue aislada de los fragmentos del nido en bandejas de fondo blanco mediante la técnica de flotación en agua, la cual permite que los individuos sean extraídos utilizando un colador de malla fina.

Una vez obtenida y depurada la población, mediante lavados consecutivos, los ejemplares se colocaron en frascos de tamaño apropiado conteniendo el líquido fijador (FAA).

Cabe aclarar que si bien esta metodología no toma en consideración la población que se halla fuera del termitero, o en el interior de las galerías y pasadizos no visibles, al momento de extraer las colonias, permite obtener la mayoría de los individuos de la colonia. Por otra parte, debido a que parte de los huevos se pierden durante la extracción de los nidos, éstos no fueron contabilizados.

A continuación, para cada una de las colonias, se procedió a:

a) Mezclar todos los individuos de una población y medir el volumen total de la misma.

b) Extraer, al azar, cinco muestras de 5 ml. cada una.

c) Separar las termites de cada muestra por categoría (obreras mayores y menores, soldados, imagos, inmaduros blancos, soldados blancos, pre-alados).

d) Contar por categoría las termites de cada muestra.

Con los datos obtenidos se calculó el promedio de individuos presentes en las muestras de $5 \mathrm{ml}$, y con ese valor se estimó la población total de la colonia y las proporciones de cada categoría.

Se utilizó el coeficiente de correlación por rangos de Spearman para determinar las correlaciones entre castas, entre el volumen de los termiteros y el volumen de las poblaciones que albergan, y entre la cantidad total de individuos estimados y el volumen de las poblaciones en cada colonia. Las diferencias entre estaciones en el tamaño de los nidos y proporciones de las diferentes castas fueron analizadas mediante el análisis de la varianza no paramétrico de Kruskall-Wallis. Todos los análisis estadísticos fueron realizados utilizando el software Statistica (STATSOFT, Inc., 1999) $\operatorname{con} \alpha=0,05$. 


\section{RESULTADOS Y DISCUSIÓN}

En la Tabla 1 se muestran los meses y lugares donde fueron recolectados los 16 nidos analizados, junto a su correspondiente volumen de población, volumen del termitero y población total estimada.

Con respecto a las dimensiones de los nidos, la altura y diámetro de los mismos registraron un valor promedio de $31,25 \mathrm{~cm}$ (mínimo $17 \mathrm{~cm}$ y máximo $58 \mathrm{~cm}$ ) y 25,48 cm (mínimo 19,4 cm y máximo 35,6 cm) respectivamente. El volumen promedio de los termiteros analizados tuvo un valor de $21947,85 \mathrm{~cm}^{3}$, variando entre $7580,232 \mathrm{~cm}^{3}$ y $43422,56 \mathrm{~cm}^{3}$. No se registraron diferencias significativas entre los volúmenes de los nidos pertenecientes a las cuatro estaciones $(\mathrm{H}=2,139 \mathrm{p}=0,543)$.

El volumen de las poblaciones correspondientes a los 16 nidos analizados, presentó un valor promedio de 147,19 ml, variando entre $50 \mathrm{ml}$ y $350 \mathrm{ml}$.

La población total estimada para cada nido se presenta en la Tabla 2 que osciló entre 5.094 y 39.834 individuos.

No se registraron diferencias significativas entre las estaciones en el volumen promedio de población, y entre el número de individuos de las poblaciones estimadas $(\mathrm{H}=2,975 \mathrm{p}=0,395$ y $\mathrm{H}=4,257 \mathrm{p}=$ 0,235 respectivamente).

Las correlaciones entre el volumen de los termiteros y la población estimada y entre el volumen de los termiteros y el volumen total de las poblaciones no fueron significativas $\left(r_{s}=0,336 ; p=0,203\right.$ y $r_{s}=0,421$; $p=0,104$ respectivamente). En cambio, sí se registró una correlación significativa entre la población estimada y el volumen de la población $\left(\mathrm{r}_{\mathrm{s}}=0,983 ; \mathrm{p}=0,000\right)$.

Tabla 1. Procedencia y fechas de extracción de las 16 colonias de Cortaritermes fulviceps. Volúmenes de las poblaciones, de los termiteros y población total estimada obtenida de cada uno de ellos.

\begin{tabular}{ccccccccc}
\hline NIDO & Mes & Estación & $\begin{array}{c}\text { Lugar de } \\
\text { procedencia }\end{array}$ & $\begin{array}{c}\text { Vol. } \\
\text { Población } \\
(\mathbf{m l})\end{array}$ & $\begin{array}{c}\text { Vol. } \\
\text { Termitero } \\
\left(\mathbf{c m}^{\mathbf{3}}\right)\end{array}$ & $\begin{array}{c}\text { Población } \\
\text { total } \\
\text { estimada }\end{array}$ & $\begin{array}{c}\text { Altura } \\
(\mathbf{c m})\end{array}$ & $\begin{array}{c}\text { Diámetro } \\
(\mathbf{c m})\end{array}$ \\
\hline $\mathbf{1}$ & Junio & Otoño & El Perichón & 250 & 43422,56 & 27740 & 58 & 26,8 \\
$\mathbf{2}$ & Junio & Otoño & El Perichón & 170 & 29197,93 & 19244 & 39 & 26,8 \\
$\mathbf{3}$ & Junio & Otoño & El Perichón & 320 & 16433,28 & 39834 & 20 & 28 \\
$\mathbf{4}$ & Junio & Otoño & El Perichón & 55 & 7857,161 & 6679 & 17 & 21 \\
$\mathbf{5}$ & Julio & Invierno & Mburucuyá & 350 & 35935,91 & 32816 & 27 & 35,6 \\
$\mathbf{6}$ & Agosto & Invierno & Laguna Pampín & 210 & 15792,41 & 23276 & 40 & 19,4 \\
$\mathbf{7}$ & Agosto & Invierno & Laguna Pampín & 120 & 27305,26 & 13795 & 34 & 27,6 \\
$\mathbf{8}$ & Agosto & Invierno & Laguna Pampín & 50 & 13907,17 & 5094 & 32 & 20,4 \\
$\mathbf{9}$ & Noviembre & Primavera & Laguna Pampín & 55 & 10002,25 & 6261 & 21 & 21,4 \\
$\mathbf{1 0}$ & Noviembre & Primavera & Laguna Pampín & 190 & 7580,232 & 22222 & 18 & 20 \\
$\mathbf{1 1}$ & Noviembre & Primavera & Laguna Pampín & 150 & 20759,85 & 17076 & 33 & 24,6 \\
$\mathbf{1 2}$ & Noviembre & Primavera & Laguna Pampín & 100 & 26359,24 & 10372 & 30 & 29 \\
$\mathbf{1 3}$ & Enero & Verano & Laguna Pampín & 60 & 23176,67 & 5359 & 35 & 25,2 \\
$\mathbf{1 4}$ & Enero & Verano & Laguna Pampín & 50 & 14800,06 & 5494 & 21 & 26 \\
$\mathbf{1 5}$ & Febrero & Verano & Av.Maipú y Ruta 12 & 135 & 32022,61 & 10568 & 46 & 25,8 \\
$\mathbf{1 6}$ & Febrero & Verano & Av.Maipú y Ruta 12 & 90 & 26612,94 & 9554 & 29 & 29,6 \\
\hline
\end{tabular}


Tabla 2. Categorías y total de los individuos contabilizados en las muestras procedentes de las 16 colonias analizadas de Cortaritermes fulviceps.

\begin{tabular}{cccccccc}
\hline Nido & $\begin{array}{c}\text { Ob. } \\
\text { Mayores }\end{array}$ & $\begin{array}{c}\text { Ob. } \\
\text { Menores }\end{array}$ & Soldados & $\begin{array}{c}\text { Inm. } \\
\text { Blancos }\end{array}$ & $\begin{array}{c}\text { Pre } \\
\text { alados }\end{array}$ & $\begin{array}{c}\text { Sol. } \\
\text { Blancos }\end{array}$ & Total \\
\hline 1 & 12640 & 6950 & 2260 & 4240 & 1650 & 0 & 27740 \\
2 & 8806 & 4332 & 2706 & 2380 & 1020 & 0 & 19244 \\
3 & 15936 & 9843 & 6182 & 7258 & 614 & 0 & 39834 \\
4 & 2702 & 2757 & 928 & 275 & 18 & 0 & 6679 \\
5 & 14014 & 14910 & 2660 & 784 & 448 & 0 & 32816 \\
6 & 7585 & 4108 & 1772 & 8476 & 1336 & 0 & 23276 \\
7 & 5630 & 3994 & 1728 & 2429 & 48 & 24 & 13795 \\
8 & 2260 & 1216 & 1556 & 35 & 34 & 0 & 5094 \\
9 & 2616 & 513 & 1173 & 130 & 0 & 730 & 6261 \\
10 & 7007 & 4241 & 4264 & 5495 & 38 & 1208 & 22222 \\
11 & 5814 & 6030 & 3018 & 2130 & 0 & 105 & 17076 \\
12 & 4960 & 3076 & 1456 & 800 & 70 & 52 & 10372 \\
13 & 1159 & 2066 & 422 & 1651 & 12 & 55 & 5352 \\
14 & 1670 & 1504 & 672 & 1576 & 0 & 72 & 5490 \\
15 & 3483 & 2560 & 826 & 3267 & 394 & 38 & 10557 \\
16 & 1822 & 842 & 450 & 5022 & 1138 & 281 & 9540 \\
\hline
\end{tabular}

Con respecto a la composición de las colonias, en $7(43,75 \%)$ de los 16 nidos analizados se encontraron individuos localizó la pareja real y en 4 solo la reina. La longitud total de las reinas varió entre 12,6 mm y $15,4 \mathrm{~mm}$ de longitud (Tabla 3 ). reproductores activos. En 3 de ellos se

Tabla 3. Presencia y mediciones de la longitud total del cuerpo de las reinas.

\begin{tabular}{cccc}
\hline Nido & Reina & Rey & Medida de Reina \\
\hline 1 & $*$ & $*$ & \\
2 & $*$ & $*$ & $14,8 \mathrm{~mm}$ \\
3 & $*$ & & $15,4 \mathrm{~mm}$ \\
4 & $*$ & & $12,6 \mathrm{~mm}$ \\
5 & $*$ & & \\
6 & & & \\
7 & & & \\
8 & & & \\
9 & & & $15,4 \mathrm{~mm}$ \\
10 & $*$ & & \\
11 & $*$ & & \\
12 & & & \\
13 & & & \\
14 & & & \\
16 & & & \\
\hline
\end{tabular}


No se registraron relaciones significativas entre el tamaño de las reinas y el volumen de la población $\left(\mathrm{r}_{\mathrm{s}}=0,36\right.$ $\mathrm{p}=0,553)$; en cambio sí resulto significativa con el volumen del termitero $\left(r_{s}=0,87\right.$ $\mathrm{p}=0,05$ ).

Las obreras, soldados e inmaduros blancos estuvieron presentes en todas las colonias analizadas, en tanto que los soldados blancos y pre-alados solo se hallaron presentes en algunas de ellas (Tabla
2). Los alados no se registraron en ninguno de los nidos analizados.

En las 4 estaciones existieron diferencias significativas en cuanto a las proporciones contabilizadas en todas las castas menos en las obreras menores (Tabla 4). Pudo observarse que obreras mayores, menores, soldados y soldados blancos disminuyen en verano mientras que los inmaduros blancos y pre-alados aumentan en esta estación (Figura 1).

Tabla 4. Significancias de las diferencias en el conteo de individuos para las 4 estaciones del año obtenidas con el análisis de Kruskall-Wallis.

\begin{tabular}{ccc}
\hline CASTAS & $\mathbf{H}(\mathbf{g l}=\mathbf{3})$ & $\mathbf{p}$ \\
\hline OB. MAYORES & 42,804200 & 0,0000 \\
OB. MENORES & 6,760000 & 0,0799 \\
SOLDADOS & 37,548250 & 0,0000 \\
INM. BLANCOS & 25,257780 & 0,0000 \\
SOL. BLANCOS & 67,321430 & 0,0000 \\
PRE ALADOS & 28,006340 & 0,0000 \\
TOTAL & 21,210230 & 0,0000 \\
\hline
\end{tabular}

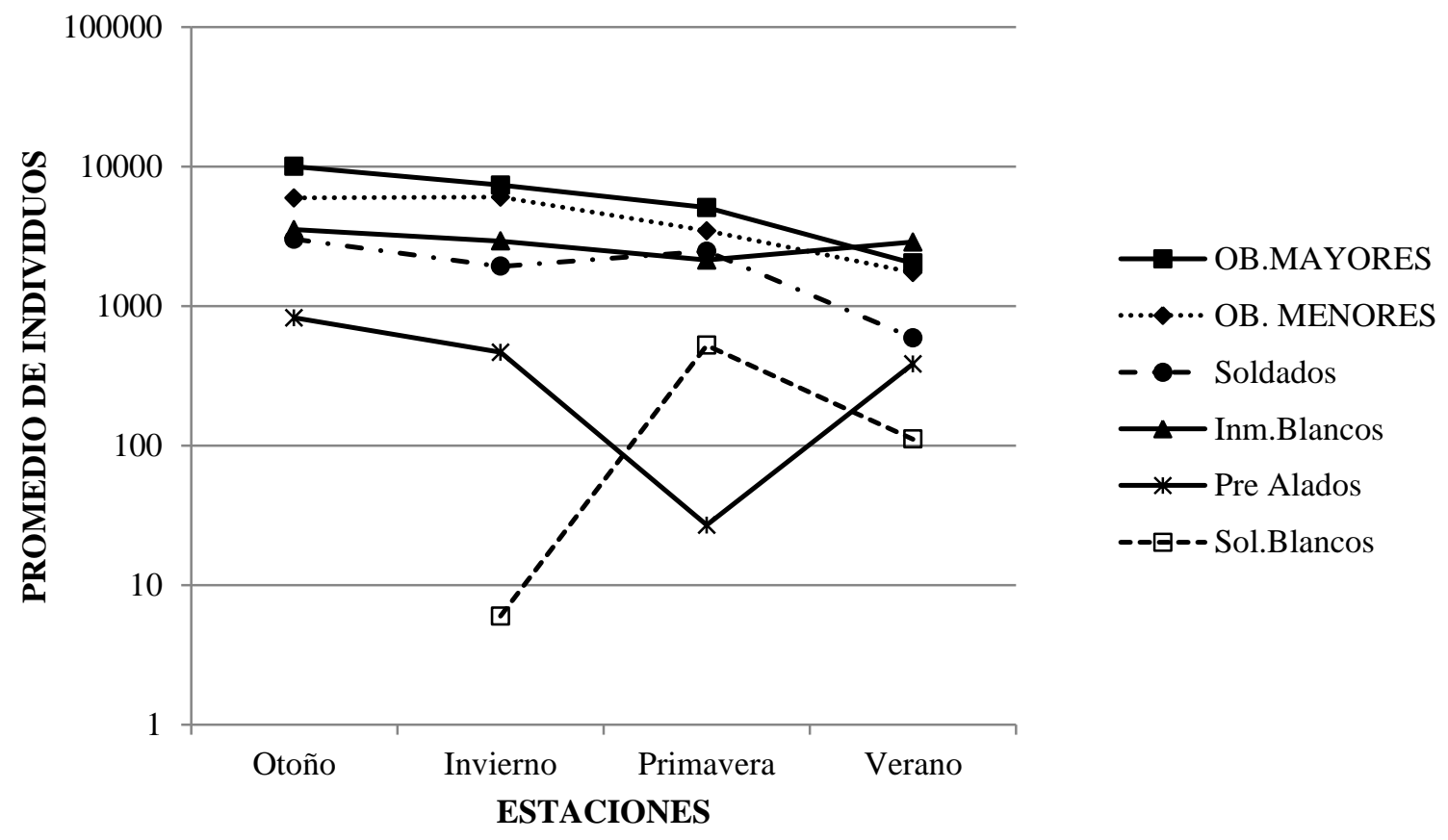

Figura 1. Variación de las castas en las cuatro estaciones del año. 
El porcentaje de obreras con respecto al número total de individuos contabilizados, varió entre $27,88 \%$ y $88,14 \%$. Los soldados en cambio, representaron entre el $4,71 \%$ y $30,55 \%$ de las termites de cada nido. Dichos valores correspondieron a las estaciones de verano e invierno respectivamente en ambas castas.

La razón del total de obreras:soldados se mantuvo constante durante todo el año con una proporción de 6:1, excepto en primavera donde se registró un descenso a $3: 1$. Se registró correlación positiva y significativa entre el total de obreras y soldados $\left(r_{s}=0,235 ; p=0,037\right)$ y entre obreras mayores y soldados $\left(r_{s}=0,379 ; p=\right.$ 0,001); en cambio no hubo correlación entre obreras menores y soldados $\left(\mathrm{r}_{\mathrm{s}}=-0,008 \mathrm{p}=\right.$ 0,944).

Con respecto a la cantidad de inmaduros blancos el máximo de individuos se observó en verano, presentando una correlación significativa negativa con obreras y soldados ( $r s=-0,729 ; p=0,000 y$ $\mathrm{rs}=-0,411 ; \mathrm{p}=0,000$ respectivamente).

Los soldados blancos se localizaron en un $56,25 \%$ de los nidos y presentándose una correlación positiva con los soldados blancos ( $r s=0,261 ; p=0,020)$.

En el 81,25\% de los nidos, estuvieron presente los pre-alados. Existió una correlación positiva entre inmaduros blancos $\mathrm{y}$ pre-alados $\left(\mathrm{r}_{\mathrm{s}}=0,552 \mathrm{p}=0,000\right)$, una correlación negativa para el total de obreras y pre-alados $\left(\mathrm{r}_{\mathrm{s}}=-0,416 ; \mathrm{p}=0,000\right)$ como así también entre soldados y pre-alados $\left(\mathrm{r}_{\mathrm{s}}=\right.$ 0,46; $\mathrm{p}=0,000)$.

En C. fulviceps las colonias analizadas tuvieron una organización social de tipo monogínica. Esto concuerda con lo encontrado por Thorne (1982) para Termes hospes (Sjostedt) y T. kraepelinii (SILVESTRI, 1903); y con C. cumulans (TORALES et al., 1999) en cambio no concuerda con lo observado por Aber (1989) para Termes saltans quien ha encontrado mas de una pareja real por nido.
Según lo establece Laffitte de Mosera et al. (1979) en su estudio sobre $C$. fulviceps, la pareja real no tiene celda o cámara real o una posición fija en el nido, generalmente se la localiza en la region hipógea, donde la población es mas abundante; o en las regiones centrales de la parte epígea del nido. Estos autores hacen referencia al hallazgo de 27 reinas en un solo nido. En los nidos analizados en este estudio los reproductores encontrados se localizaron en la parte hipógea y en todos los casos se encontro una reina o pareja real.

La presencia de obreras y soldados en las cuatros estaciones del año fue constante, esto es habitual en varias especies de termites, tales como $C$. fulviceps (LAFFITTE DE MOSERA et al., 1979) en Uruguay, y en el nordeste de Argentina $C$. cumulans (TORALES et al., 1999), $T$. saltans (CORONEL et al., 2001), M. strunckii (TORALES \& CORONEL, 2004), $N$. coxipoensis (TORALES et al., 2006) y $N$. aquilinus (ETCHEVERRY et al., 2010). De igual manera, Aber (1989) registra un resultado similar para $T$. saltans de Uruguay.

La razón obreras:soldados varía con las diferentes especies, así para poblaciones de Termes saltans de Uruguay Aber (1989) encuentra que permanece constante todo el año con un valor de 90:1; en cambio en estudios realizados con poblaciones de esta misma especie en la Provincia de Corrientes Coronel et al. (2001) encuentran que la razón obreras:soldados varía entre 39:1 en primavera y 64:1 en invierno. En el presente estudio, C. fulviceps mostró una razón de 6:1 que se mantuvo constante durante todo el año, a excepción de primavera donde se registró un descenso a 3:1, este último valor coincide con lo obtenido para la misma especie en Uruguay por Laffitte de Mosera et al. (1979) quienes manifiestan que este valor se mantiene constante durante todo el año.

La presencia de inmaduros blancos durante todo el año indicaría, tal como 
sugiere Torales et al. (1999), que el invierno poco riguroso de nuestra región permite que la puesta de huevos por parte de los reproductores primarios o de reemplazo sea ininterrumpida a lo largo del año. Este mismo resultado fue observado en otras termites del nordeste argentino como $T$. saltans (CORONEL et al., 2001), $M$. strunckii (TORALES \& CORONEL, 2004), C. cumulans (TORALES et al., 1999) y $N$. aquilinus (ETCHEVERRY et al., 2010).

En algunas especies de termites como Trinervitermes ebenerianus Sjostedt y $T$. saltans (CORONEL et al., 2001) se han registrado un comportamiento antagónico en las proporciones de obreras e inmaduros blancos, en estas especies el descenso de la cantidad de obreras en verano es coincidente con el aumento en el número de inmaduros blancos y podría deberse al recambio de generaciones que ocurren generalmente al momento de producirse los enjambramientos, en este estudio sin embargo las proporciones de obreras e inmaduros blancos parecen estar sincronizadas a lo largo del año.

Los soldados blancos solamente se encontraron en colonias pertenecientes a primavera y verano, tal como ocurre con $C$. cumulans (TORALES et al., 1999), $M$. strunckii (TORALES \& CORONEL, 2004) y $N$. coxipoensis (TORALES et al., 2006), sin embargo Etcheverry et al. (2010) para colonias de $N$. aquilinus, los hallaron en mayor cantidad en verano y otoño. Esto nos estaría sugiriendo que los soldados no se generan de manera constante dentro de las colonias (DARLINGTON, 1991) sino en relación al vuelo imaginal (TORALES et al., 1999) por la necesidad de reemplazar los soldados que mueren al exponerse a eventuales depredadores durante el éxodo de los alados (HOWARD \& HAVERTY, 1981).

La diferencia significativa encontrada en este trabajo para las obreras mayores, soldados, inmaduros blancos, soldados blancos y pre-alados con respecto a las cuatro estaciones, es similar a lo hallado por Coronel et al. (2001) para T. saltans. La mayor cantidad de pre-alados estuvieron presentes en las estaciones de verano, otoño e invierno, las ninfas no siempre se encontraron en todos los nidos durante el período que corresponde a su desarrollo, debido quizás a factores internos de cada colonia (TRUCKENBRODT, 1978). En los nidos analizados nunca se los encontró junto a los alados, tal como aparecen citados en algunos trabajos para especies constructoras de montículos tales como $C$. fulviceps (LAFFITTE DE MOSERA et al., 1979), $C$. cumulans (TORALES et al., 1999) y $T$. saltans (ABER, 1989). La ausencia de alados en todos los muestreos podría deberse a la senectud de los nidos (BODOT, 1969) o al hecho de que las colectas se realizaron con posterioridad al proceso de enjambramiento.

En la mayor parte de las especies tropicales, las ninfas maduran en un lapso de cinco a siete meses; luego de dicha maduración suele transcurrir un tiempo antes del vuelo nupcial para fundar una nueva colonia (LEPAGE, 1983; NOIROT, 1990). La época de enjambramiento se estima que ocurre entre Octubre y Enero, lo cual se infiere por el hallazgo de pre-alados a fines de invierno que poseían un mayor desarrollo de sus esbozos alares y además porque fue en primavera donde se registró la menor cantidad de esta casta. Esta situación ya fue mencionada por Laffitte de Mosera et al. (1979) para C. fulviceps en Uruguay quien encuentra que el enjambramiento ocurre entre octubre y noviembre, continuando hasta enero. Para $N$. coxipoensis en la provincia de Corrientes se produce en noviembre (TORALES et al., 2006); para $C$. cumulans, a finales del invierno o comienzo de la primavera (TORALES et al., 1999); para M. strunckii (MILL, 1983) la aparición de los alados se da en agosto; Procornitermes striatus (Hagen, 1958) presenta la aparición de alados entre 
septiembre y octubre (BENTOS DE ODRIOZOLA, 1985 citado en ABER, 1989); mientras que para $N$. aquilinus (ETCHEVERRY et al., 2010), ocurre entre agosto y octubre.

La falta de correlación encontrada entre el volumen de los nidos y el de la población total estimada para esta especie, concuerda con lo analizado anteriormente para $N$. coxipoensis (TORALES et al., 2006) y para $N$. aquilinus (ETCHEVERRY et al., 2010), esto probablemente se deba a que la metodología utilizada en estos estudios no toma en consideración todos aquellos individuos que se encuentran fuera del nido al momento de extraerlos.

La relación positiva registrada para el total de la población estimada y el volumen de población, es similar a lo hallado por Etcheverry et al. (2010) para N. aquilinus.

\section{CONCLUSIONES}

La organización de la colonia de $C$. fulviceps es aparentemente, de acuerdo a lo observado, de tipo monogínica. La oviposición se registró durante todo el año. Obreras y soldados estuvieron presentes en las cuatro estaciones. Proporcionalmente, la casta de obreras superó a la de soldados en las 4 estaciones. Los soldados blancos solo estuvieron presentes en las estaciones primavera y verano; en cambio los inmaduros blancos estuvieron presentes durante todo el año. Además hubo diferencias significativas en cantidad entre las 4 estaciones para obreras mayores; soldados; inmaduros blancos; soldados blancos y pre-alados. La mayor cantidad de pre-alados se registró en las estaciones de verano, otoño e invierno. La época de enjambramiento se estima que es entre los meses de octubre a enero. Si bien muchos aspectos de la biología de $C$. fulviceps son conocidos, ésta es la primera vez que se brinda información sobre el tamaño de las colonias, las proporciones de las diferentes castas dentro de los nidos, y las variaciones estacionales en las cantidades de individuos de cada casta. Estos constituyen un considerable aporte al conocimiento poblacional de una de las especies constructora de montículos mejor representada en el nordeste de la República Argentina y países limítrofes.

\section{AGRADECIMIENTOS}

A la secretaria General de Ciencia y Técnica de la Universidad Nacional del Nordeste por el financiamiento del proyecto F-015 "Bio-ecología de termites (Insecta, Isoptera) del Parque Nacional Mburucuyá (Corrientes, Argentina)" en el marco del cual se realizó este estudio.

\section{REFERENCIAS BIBLIOGRAFICAS}

ABER, A. 1989. Análisis de la población de los nidos de Termes saltans (Wasmann, 1897) (Isoptera, Termitidae) en Uruguay: Ciclo anual de castas y enjambrazón. Revista Brasileira de Entomologia, 33 (2): 161-168.

ANNONI, G.; CORONEL, J.M.; LAFFONT, E.R. 2011. Análisis morfométrico de la casta obrera de Cortaritermes fulviceps (Isoptera, Termitidae). Revista de Agricultura, n. 86 (3): 200-206.

BAGINE, R.K.N.; DARLINGTON, J.E.P.C.; KAT, P.; RITCHIE, J.M. 1989. Nest structure, populations structure and genetic differentiation of some morphologically similar species of Macrotermes in Kenya. Sociobiology, 15: $125-132$.

BARONI-URBANI， C.; JONSENS， G.; PEAKIN, G. J. 1978. Empirical data and demografic parameters. (págs. 5-44). En: Brian, M. V. (ed), Production Ecology of Ants and Termites. Cambridge University Press. Cambridge. 409 p. 
BODOT, P. 1969. Composition des colonies de termites: ses fluctuations au cours du temps. Insectes Sociaux, 16 (1): 39-54.

CONSTANTINO, R. 1998. Catalog of the living termites of the New World. (Insecta: Isoptera). Arquivos de Zoología, n. 35 (2): 135-231.

CORONEL, J.M.; LAFFONT, E.R; TORALES, G.J.; PORCEL, E.. 2001. Variación estacional en la composición de colonias de Termes saltans Wasmann (Isoptera: Termitidae, Termitinae). Facena, 17: 3-13.

CORONEL, J.M.; LAFFONT, E.R. 2010. Disposición espacial de Cortaritermes fulviceps (Isoptera, Termitidae, Nasutitermitinae) en una localidad de la Provincia de Corrientes, Argentina. Revista de Agricultura, n.85 (1): 6167.

DARLINGTON, J. 1984. A method for sampling the populations of large termite nests. Annals of Applied Biology, 104: 427-436.

DARLINGTON, J.P. 1991. Turnover in the populations within mature nests of the termite Macrotermes michaelseni in Kenya. Insectes Sociaux, 38: 251-262.

ETCHEVERRY, C.; GODOY, M.C.; TORALES, G.J. 2010. Dinamica Poblacional de Nasutitermes aquilinus (Insecta, Isoptera; Termitidae) en la Provincia de Corrientes (Argentina). Facena, 26: 15-27.

HAVERTY, M.I.; NUTTING, W.L. 1975. Density, Dispersion, and Composition of Desert Termite foraging Populations and their Relationship to Superficial Dead Wood. Environmental Entomology, 4 (3): 480-486.

HOWARD, R.; HAVERTY, M.I. 1981. Seasonal variation in caste proportions of fields colonies of Reticulitermes flavipes (Kollar). Environmental Entomology, 10 (4): 546-549.

JOUQUET, P.; BOULAIN, N.; GIGNOUX, J.; LEPAGE, M. 2004. Association between subterranean termites and grasses in a West African savanna: spatial pattern analysis shows a significant role for Odontotermes $n$. pauperans. Applied Soil Ecology 27: 99-107.

KORB, J.; LINSENMAIR, K.E.. 2001. The causes of spatial patterning of mounds of a fungus - cultivating termite: results from nearest-neighbour analysis and ecological studies. Oecologia 127: 324333.

LAFFITTE DE MOSERA, S.; TALICE, R. V.; DE SPRECHMANN, A.M.S.; ABER DE STERZMAN, A. 1979. Estudio poblacional anual de Nasutitermes fulviceps (Silvestri, 1901). Revista de Biología del Uruguay 7 (2): 77-87.

LEFEUVE, P. 1987. Replacement queens in the neotropical termite Nasutitermes coxipoensis. Insectes Sociaux 34 (1): 10-19.

LEPAGE, M. 1983. Structure et dynamique des peuplements de termites tropicaux. Acta Oecologica 4 (1): 65-87.

MARTIUS, C. 1994. Diversity and ecology of termites in Amazonian forests. Pedobiologia 38: 407-428.

MILL, A.E. 1983. Generic keys to the soldier caste of New World Termitidae (Isoptera: Insecta). Systematic Entomology 9: 179-190.

NOIROT, C. 1990. Sexual castes and reproductive strategies in termites. (págs. 5-31). En: Engels, W. (ed.) Social Insects Berling: Springer-Verlag.

REDFORD, K.H. 1984. The termitaria of Cornitermes cumulans (Isoptera, Termitidae) and their role in determining a potential keystone species. Biotropica 16 (2): 112-119.

SILVESTRI, F. 1903. Contribuzione alla conozcenza dei termitidi e termitofili dell'America Meridionale. Redia 1: 1234. 
STATSOFT, INC. 1999. STATISTICA for Windows. Tulsa. Disponible en : <http://www.statsoft.com>

TALICE, R.V.; LAFFITTE DE MOSERA, S.; CAPRIO, R.; DE SPRECHMANN A.M.S. 1969. Estructura de los termiteros de Nasutitermes fulviceps (Silvestri, 1901). Publicaciones del Departamento de Biología General y Experimental. Facultad de Humanidades y Ciencias. Montevideo, Uruguay, 2: 1-20.

THORNE, B.L. 1982. Polygyny in Termites: Multiple primary queens in colonies of Nasutitermes corniger (Motschuls) (Isoptera, Termitidae). Insectes Sociaux, 29 (1): 102-117.

THORNE, B.L. 1985. Numerical and Biomass caste proportions in colonies of the termites Nasutitermes corniger and $N$. ephratae (Isoptera: Termitidae). Insectes Sociaux, 32 (4): 411-426.

TORALES, G.J. 1998. Isoptera. (págs. 4866). En: Biodiversidad de Artópodos Argentinos. Una perspectiva biotaxonómica. Coscarón, S. y J.J. Morrone (Directores). Ediciones Sur, La Plata, Argentina. 559 p.

TORALES, G.J.; CORONEL, J.M. 2004. Qualitative and Quantitative composition of colonies of Microcerotermes strunckii (Isoptera: Termitidae). Sociobiology, 43 (3): 523534.

TORALES, G.J.; LAFFONT, E.R.; ARBINO, M.O.; CORONEL, J.M.. 1999. Composición de colonias de Cornitermes cumulans (Kollar) (Isoptera: Termitidae, Nasutitermitinae) en diferentes épocas del año. Revista de la Sociedad Entomológica Argentina. 58 (3-4): 189-196.

TORALES, G.J.; LAFFONT, E.R.; GODOY, M.C.; CORONEL, J.M.; ARBINO, M.O. 2005. Update on Taxonomy and Distribution of Isoptera from Argentina. Sociobiology, 45 (3): 853-886.

TORALES, G.J.; CORONEL, J.M.; LAFFONT, E.R.; GODOY, M.C.; ARBINO, M.O.; PORCEL, E. 2006. Analysis of the population from Nasutitermes coxipoensis nests (Isoptera: Termitidae, Nasutitermitinae), from a locality of the Province of Corrientes (Argentina). Sociobiology, 48 (1): 223-236.

TORALES, G.J.; CORONEL, J.M.; GODOY, M.C.; LAFFONT, E.R.; ROMERO, V.L. 2008. Additions to the Taxonomy and Distribution of Isoptera from Argentina. Sociobiology, 51 (1): 31-47.

TRUCKENBRODT, W. 1978. About the time and place of appearance and the number of nymphs of Odontotermes stercorivorus ( $\mathrm{Sj}$.$) (Insecta, Isoptera).$ Insectes Sociaux, 25 (4): 303-313.

Recebido em: 26/03/2013 Aceito para publicação em: 18/08/2013 